\title{
Pyruvate kinase M2 and its evolving role as a prognostic marker in systemic malignancies besides colorectal carcinomas
}

\author{
Shailendra Kapoor
}

Received: 16 August 2012 / Accepted: 17 September 2012 /Published online: 4 October 2012

(C) International Society of Oncology and BioMarkers (ISOBM) 2012

To the editor:

I read with great interest the recent article by Cantillo et al. [1]. Interestingly, recent data suggest that tumor M2-pyruvate kinase may influence prognosis in a number of systemic malignancies besides colon carcinomas.

For instance, there is a close co-relationship between microvessel density as well as tumor prognosis in biliary tract carcinomas and biliary M2-pyruvate kinase levels. Using enzymelinked immunosorbent assay, biliary M2-pyruvate kinase has a sensitivity of nearly $90 \%$ for identifying biliary tract carcinomas [2]. Similarly, tumor M2-pyruvate kinase plays a role in the evolution of gastric carcinomas. It does this by modulating $\mathrm{p} 65$ and Bcl-xL function [3]. Accentuated tumor expression by gastric carcinomas usually points to a poor clinical outcome and a worse prognosis. Higher tumor M2-pyruvate kinase levels are also seen in pancreatic malignancies and may help to distinguish aggressive exocrine tumor types from benign chronic pancreatitis [4]. The sensitivity of tumor M2-pyruvate kinase as a tumor marker in pancreatic malignancies is similar to CA 19.9 and averages to about $95 \%$.

Chemotherapeutic agents such as cyclosporine attenuate tumor M2-pyruvate kinase expression and thereby mitigate tumor growth in breast malignancies in a dose-dependent manner [5]. Tumor M2-pyruvate kinase can also be used in assessing prognosis in cervical carcinomas. For instance, the stage of the cervical malignancy correlates closely with tumor M2pyruvate kinase levels [6]. A similar relationship between tumor prognosis and tumor M2-pyruvate kinase levels is seen in pulmonary malignancies. In fact, use of agents such as cisplatin in conjunction with tumor M2-pyruvate kinase interfering RNA results in enhanced apoptosis and attenuates tumor growth in pulmonary malignancies [7]. The tumor volume is markedly reduced following administration of the combination.

The above examples clearly illustrate the evolving role of tumor M2-pyruvate kinase in oncology and the need for further studies to fully elaborate its prognostic role in other gastrointestinal malignancies.

\section{References}

1. Fatela-Cantillo D, Fernandez-Suarez A, Moreno MA, Gutierrez JJ, Iglesias JM. Prognostic value of plasmatic tumor M2 pyruvate kinase and carcinoembryonic antigen in the survival of colorectal cancer patients. Tumour Biol. 2012;33(3):825-32.

2. Dhar DK, Damink SW, Brindley JH, et al. Pyruvate kinase M2 is a novel diagnostic marker and predicts tumor progression in human biliary tract cancer. Cancer. 2012. doi:10.1002/cncr.27611.

3. Kwon OH, Kang TW, Kim JH, et al. Pyruvate kinase M2 promotes the growth of gastric cancer cells via regulation of Bcl-xL expression at transcriptional level. Biochem Biophys Res Commun. 2012;423:38-44.

4. Novotny I, Dite P, Dastych M, et al. Tumor marker M2-pyruvatekinase in differential diagnosis of chronic pancreatitis and pancreatic cancer. Hepatogastroenterology. 2008;55:1475-7.

5. Kumar Y, Gurusamy K, Pamecha V, Davidson BR. Tumor M2pyruvate kinase as tumor marker in exocrine pancreatic cancer a meta-analysis. Pancreas. 2007;35:114-9.

6. Jiang K, He B, Lai L, et al. Cyclosporine A inhibits breast cancer cell growth by downregulating the expression of pyruvate kinase subtype M2. Int J Mol Med. 2012;30:302-8.

7. Guo W, Zhang Y, Chen T, et al. Efficacy of RNAi targeting of pyruvate kinase M2 combined with cisplatin in a lung cancer model. J Cancer Res Clin Oncol. 2011;137:65-72.
S. Kapoor $(\bowtie)$

Formerly University of Illinois at Chicago,

Mechanicsville, VA 23111, USA

e-mail: shailendrakapoor@yahoo.com 